\title{
Fruit, Vegetable and Antioxidant Intakes are Lower in Older Adults with Depression
}

\author{
Martha E. Payne, PhD, RD, MPH ${ }^{1,{ }^{*}}$, Susan E. Steck, PhD, RD, MPH², Rebecca R. George, \\ $\mathrm{MSPH}^{3}$, and David C. Steffens, MD, MHS ${ }^{4}$ \\ Before 7/1/2012: Professor and Vice Chair of Education, Duke University (The Departments of \\ Psychiatry and Behavioral Sciences, and Medicine); Durham, North Carolina, USA \\ ${ }^{1}$ Associate Professor, Duke University (The Department of Psychiatry and Behavioral Sciences); \\ 2200 West Main Street, Suite B210, Durham, North Carolina, 27705, USA; phone (919) \\ 416-7543, fax (919) 416-7547; martha.payne@duke.edu \\ ${ }^{2}$ Associate Professor, University of South Carolina (The Department of Epidemiology and \\ Biostatistics); Columbia, SC, USA \\ ${ }^{3}$ Research Analyst, SC Central Cancer Registry; Columbia, SC, USA Until 5/3/2012: Graduate \\ student, University of South Carolina (The Department of Epidemiology and Biostatistics); \\ Columbia, SC, USA \\ ${ }^{4}$ Professor and Chairman, University of Connecticut (Department of Psychiatry); Farmington, CT, \\ USA
}

\section{Abstract}

Studies have shown an association between depression and both antioxidant levels and oxidant stress, but generally have not included intakes of antioxidants and antioxidant-rich fruits and vegetables. The present study examined the cross-sectional associations between clinicallydiagnosed depression and intakes of antioxidants, fruits and vegetables in a cohort of older adults. Antioxidant, fruit and vegetable intakes were assessed in 278 elderly participants (144 with depression, 134 without depression) using a Block 1998 food frequency questionnaire, which was administered between 1999 and 2007. All participants were age 60 years or over. Vitamin C, lutein and cryptoxanthin intakes were significantly lower among depressed individuals than in comparison participants $(\mathrm{p}<0.05)$. In addition, fruit and vegetable consumption, a primary determinant of antioxidant intake, was lower in depressed individuals. In multivariable models, controlling for age, sex, education, vascular comorbidity score, body mass index, total dietary fat, and alcohol, vitamin C, cryptoxanthin, fruits and vegetables remained significant. Antioxidants from dietary supplements were not associated with depression. Antioxidant, fruit and vegetable intakes were lower in individuals with late-life depression than in comparison participants. These associations may partially explain the elevated risk of cardiovascular disease among older depressed individuals. In addition, these findings point to the importance of antioxidant food sources rather than dietary supplements.

\footnotetext{
(c) 2012 Elsevier Ltd. All rights reserved.

*Corresponding Author.

Publisher's Disclaimer: This is a PDF file of an unedited manuscript that has been accepted for publication. As a service to our customers we are providing this early version of the manuscript. The manuscript will undergo copyediting, typesetting, and review of the resulting proof before it is published in its final citable form. Please note that during the production process errors may be discovered which could affect the content, and all legal disclaimers that apply to the journal pertain.

This work was presented as part of the American Society for Nutrition program at Experimental Biology 2011 in Washington, D.C.
} 


\section{Keywords}

antioxidant; plant foods; depression; older adults

\section{INTRODUCTION}

Depression is a serious and common mental disorder, and a significant burden to both individuals and society. Depression afflicts 121 million people, is the third leading cause of disease burden and projected to be number one by the year 2030, and is the leading cause of disability worldwide. ${ }^{1}$ Depression is responsible for the majority of 870,000 suicides annually. ${ }^{1}$ Late-life depression, occurring after age 60 years, contributes to higher risk of dementia, heart disease, and doubles health care costs. ${ }^{2,3}$ Depression has been associated with poor nutrition, which may be both a contributor to and consequence of depression. One critical aspect of poor nutrition is inadequate consumption of fruits and vegetables, and the consequent low intake of antioxidant nutrients.

Antioxidants may protect against aging and chronic diseases, ${ }^{4,5}$ and may have a biologic role in depression etiology. Oxidative stress has been found to be higher in the depressed while antioxidant capacity is lower. ${ }^{6-8}$ One marker of oxidative damage to DNA, 8-hydroxydeoxyguanosine, is elevated in depression. ${ }^{9}$ Antidepressant treatment may lower oxidative stress and raise serum antioxidant capacity. ${ }^{10}$ Unfortunately these studies have often ignored dietary intake so it is not clear if serum changes reflected dietary changes during and after a depressive episode, or were due to effects of the illness or medications.

Higher consumption of fruits and vegetables has been found to lead to better cognitive test scores, fewer depressive symptoms, and lower risk of depression. ${ }^{11,12}$ Intakes of betacarotene, vitamin C, fiber, and folate, nutrients found in fruits and vegetables, have been shown to be lower in those with depression compared to non-depressed individuals. ${ }^{13-15}$ Lastly, while plant foods and food-derived phytochemicals have been associated with health benefits, antioxidants from dietary supplements appear to be less beneficial and may, in fact, be detrimental to health. ${ }^{16}$

The study objective was to examine the relationship between late-life depression and intakes of fruits, vegetables, and antioxidants. The authors hypothesized that fruit, vegetable, and antioxidant intakes would be lower in the depressed group as compared to a non-depressed control group. Further, the authors hypothesized that antioxidant differences would be limited to those from food sources.

\section{METHODS}

Design

This case-control study occurred within a larger longitudinal clinical examination of depression in older adults (NeuroCognitive Outcomes of Depression in the Elderly). ${ }^{17}$ Nutritional data were collected between 1999 and 2007.

\section{Sample}

All participants who completed a nutrition questionnaire were included in the current study, and were blinded to any focus on antioxidants, although they had been informed that relationships between diet and depression would be examined. This sample included patients who met DSM-IV diagnostic criteria for major depressive disorder at study baseline, and comparison participants recruited from the community. Participants were aged 60 years or older, and could speak and write English. 
Exclusion criteria included a concurrent diagnosis of a psychiatric or neurological illness, and significant cognitive impairment (as indicated by a Mini-Mental State Examination score of less than 24 out of 30). In addition, participants with severe depression symptomatology were excluded because of concerns about subject burden given the length and difficulty of the food frequency questionnaire, and memory impairment that is common with depression and could impact accuracy of the reporting. This criterion was evaluated by the treating psychiatrist on a case-by-case basis, an approach that was used because the clinician was most familiar with a patient's limitations. Comparison participants were required to have no evidence of a depression diagnosis.

After complete description of the study to the participants, written informed consent was obtained. This research protocol has been reviewed and approved by the Duke University Institutional Review Board, and was in compliance with Health Insurance Portability and Accountability Act (HIPAA) guidelines.

Treatment

Depression patients received individualized treatment from a psychiatrist, who followed them throughout the study. Most depressed participants received antidepressant medication.

\section{Measures}

Depression and Medical Comorbidity-At baseline a trained interviewer administered the Duke Depression Evaluation Schedule, which includes sections of the National Institute of Mental Health (NIMH) Diagnostic Interview Schedule which assesses depression, as well as items on self-reported physical health. ${ }^{18}$ Four conditions were included in the comorbidity score: diabetes, hypertension, arteriosclerosis, and heart trouble. Each condition was assigned a score of 1 if the condition was not reported, and 2-4 indicating the condition was reported as present and interfered not at all (code 2), interfered a little (code 3), or interfered a lot (code 4). The comorbidity score thus had a possible range from 4 to 16 .

Dietary Assessment-The nutrition protocol has been described previously. ${ }^{19}$ The 1998 Block Food Frequency Questionnaire (FFQ; NutritionQuest; Berkeley, CA) was selfadministered to assess dietary intake over the preceding year, and has been validated against other nutrition assessment instruments. ${ }^{20}$ Returned questionnaires were checked for completeness and excluded if more than 15 food items were skipped. Participants who reported improbable energy intakes ( $<500 \mathrm{kcals} /$ day or $>5000 \mathrm{kcals} /$ day) were excluded from analyses. Intake estimates include total energy, servings of fruits and vegetables, and antioxidants. Antioxidant estimates include vitamin C, vitamin E (alpha- and gammatocopherol), selenium and carotenoids (alpha-carotene, beta-carotene, beta-cryptoxanthin, lutein/zeaxanthin and lycopene). Vitamin $\mathrm{C}$, alpha-tocopherol and beta-carotene were estimated from both foods and dietary supplements, while other antioxidants were estimated only from food sources. For selenium, the FFQ provided intake estimates from both food and supplemental sources, but only supplemental intakes were evaluated as food estimates are inaccurate due to the high-variability of selenium in the soil. Body mass index $\left(\mathrm{kg} / \mathrm{m}^{2}\right)$ was calculated from self-reported weight and height at time of FFQ.

\section{Statistical Analyses}

All statistical analyses were conducted using JMP software, version 8.0 (2009, SAS Institute Inc., Cary, NC). General descriptive statistics were calculated to examine the characteristics of the sample by group (depression vs. comparison). Bivariate comparisons between group and covariates were conducted using (a) chi-squared test if the covariates were categorical (sex and race), and (b) t-test if the variables were continuous (age, education, comorbidity score, BMI, kilocalories, fat, and alcohol). Race was dichotomized (White/non-White) 
because of small sample sizes in non-White categories. To describe the depression and comparison groups in terms of intake of fruits, vegetables, and antioxidants, t-tests were performed.

Logistic regression was performed to determine the effect of fruit, vegetable and antioxidant intakes upon depression group while controlling for potential confounders. Covariates were selected by backwards stepwise regression (cutoff of $p>0.2$ for exclusion). Covariates examined in this way included age, sex, race, education, comorbidity score, BMI, kilocalories, total fat, alcohol intake and dietary supplement use.

\section{RESULTS AND DISCUSSION}

This sample of older adults included a total of 144 depression and 134 comparison participants, after the exclusion of 12 participants ( $\mathrm{n}=9$ for missing covariate data, $\mathrm{n}=3$ for energy intakes below $500 \mathrm{kcal})$. The comparison group was older $(\mathrm{t}=-2.39, \mathrm{df}=276$, $\mathrm{p}=0.0175)$, included more women $(\chi 2=8.26, \mathrm{df}=1, \mathrm{p}=0.0040)$, had more years of education $(\mathrm{t}=-3.63, \mathrm{df}=276, \mathrm{p}=0.0003)$, a lower comorbidity score $(\mathrm{t}=5.03, \mathrm{df}=276, \mathrm{p}<0.0001)$, and lower BMI $(\mathrm{t}=4.21, \mathrm{df}=276, \mathrm{p}<0.0001)$ than the depression group (Table 1$)$. In addition, the comparison group consumed more alcohol $(\mathrm{t}=-2.52, \mathrm{df}=276, \mathrm{p}=0.0123)$ and less fat $(\mathrm{t}=$ $2.63, \mathrm{df}=276, \mathrm{p}=0.0089$ ). Regarding antioxidant foods and nutrients, the depression group reported a lower intake of fruits $(\mathrm{t}=-4.22, \mathrm{df}=276, \mathrm{p}<0.0001)$, vegetables $(\mathrm{t}=-2.15, \mathrm{df}=276$, $\mathrm{p}=0.0323)$, beta-cryptoxanthin $(\mathrm{t}=-4.18, \mathrm{df}=276, \mathrm{p}<0.0001)$, lutein $(\mathrm{t}=-2.29, \mathrm{df}=276$, $\mathrm{p}=0.0227)$, and vitamin $\mathrm{C}$ from foods $(\mathrm{t}=-5.10, \mathrm{df}=276, \mathrm{p}<0.0001)$ (Table 2$)$.

Separate logistic regression models were run for fruits, vegetables, and each antioxidant variable (Table 3). Fruits, vegetables, vitamin $\mathrm{C}$ from foods, and beta-cryptoxanthin were significantly lower in depressed group, as in the bivariate analyses, while lutein was no longer significant.

Overall, this study showed lower intakes of fruits, vegetables, vitamin $C$, and $\beta$ cryptoxanthin in older adults with depression as compared to non-depressed comparison participants. Only food sources of antioxidants were inversely associated with depression. This may indicate that the form and delivery of the antioxidants are important, and that other components of fruits and vegetables may be beneficial for depression.

This study is consistent with prior work showing that depressed individuals are less likely to consume healthy diets than are non-depressed individuals. ${ }^{11,19,21}$ One prior study showed that only naturally-occurring food sources of folate were associated with depression while folic acid from fortification and dietary supplements was not, again indicating that a diet rich in plant foods may be beneficial. ${ }^{15}$ Consistent with this idea, several studies have found that dietary patterns rich in fruits and vegetables were associated with lower risk of depression. ${ }^{11,12,22-24}$

In terms of specific antioxidants, only vitamin $\mathrm{C}$ and beta-cryptoxanthin were lower in depressed elders. A previous study found that dietary changes which included increasing vitamin $\mathrm{C}$ intakes were associated with a decrease in depression symptoms. ${ }^{25}$ Older adults with depression also have lower serum levels of vitamin C. ${ }^{26}$ Regarding beta-cryptoxanthin, this finding may relate to the unique carotenoid profile of the brain. Xanthophylls concentrations are disproportionately high in the brain, compared to other organs such as the retina, and beta-cryptoxanthin is the predominant xanthophyll in the brains of older adults. ${ }^{27}$ In addition, beta-cryptoxanthin concentrations in the frontal lobe, but not occipital lobe, decline with age. This may be particularly important for late-life depression given that dysfunction of the frontal lobe is considered central to its etiopathology. ${ }^{28,29}$ 
One potential mechanism to explain a protective association between antioxidants and depression is that antioxidants defend against the negative effects of oxidative stress, which has been associated with depression. 6,7 The brain is especially susceptible to oxidative stress due to high levels of aerobic respiration, as well as the high content of polyunsaturated fatty acids which are susceptible to damage from reactive oxygen species. Another explanation for the antioxidant-depression relationship is that antioxidants have beneficial effects upon inflammatory markers, ${ }^{30}$ which are known to be elevated in depression. ${ }^{31}$ This idea is consistent with the InCHIANTI Study which showed that higher serum levels of carotenoids were associated with lower risk of depression, and that this effect was partially mediated by the inflammatory marker Interleukin-1 receptor antagonist. ${ }^{30}$ Alternatively, the observed associations may be due not to antioxidants but rather to other dietary factors such as folate that also occur in fruits, vegetables and plant-rich diets.

Strengths of this study include the rigorous evaluation of depression. A psychiatrist evaluated and diagnosed individuals with major depressive disorder, and then treated and followed depressed participants for the duration of the study. This is in contrast to many studies that have examined diet and depression - not only do most studies not involve a psychiatrist but depression is often indicated by self-report of one or two depression symptoms rather than by clinical diagnosis. Another strength is that participants reported antioxidant intakes similar to national norms. ${ }^{32}$

A limitation of this study is the modest sample size $(n=278)$ which may have limited power to detect associations. In addition, this study cannot confirm a causal relationship between depression and intakes of fruits, vegetables and antioxidants, given that the nutrition assessment was administered after depression diagnosis, as well as the lack of random assignment. Depression and treatments for depression, primarily antidepressants, are known to affect appetite and dietary intake. ${ }^{33,34}$ However, kilocalories were examined as a potential covariate and found to be non-significant, and there is no evidence that treatment type has effects upon antioxidant intakes. Another possible limitation is the exclusion of severelydepressed individuals from the nutrition protocol, which likely would have made it more difficult to detect differences between depression and comparison participants. An added concern is that the decision to exclude these participants was made by the treating psychiatrist rather than an assessor not involved in participant's treatment. The rationale was that the treating psychiatrist would be in the best position to determine whether participation would represent an undue burden for the subject. An alternate approach of an unaffiliated assessor might represent a more standardized and objective approach. However, it is unclear what effect this would have had upon inclusion in the study. Self-report of diet has known limitations, including potential for inaccurate subject recall, which may have differed between groups. Finally, these results may be generalizable only to older individuals of primarily European descent with depression who receive psychiatric treatment.

\section{CONCLUSIONS}

Intakes of fruits, vegetables, and naturally-occurring antioxidants, specifically vitamin $\mathrm{C}$ and beta-cryptoxanthin, were found to be inversely associated with depression in this sample of elderly depression and comparison participants, while no association was found with supplemental antioxidants. These results may indicate that components of fruits and vegetables, including specific antioxidants, are important for mental health. Antioxidants from dietary supplements may not confer the same benefits. Future studies are needed to confirm these findings, to establish an etiological relationship between antioxidants and depression, and to clarify the mechanisms by which fruits and vegetables may influence depression and brain health. If low intakes of fruits, vegetables and antioxidants are found to 
cause or exacerbate late-life depression, it may be possible to prevent depression or to lessen its negative impact through dietary intervention.

\section{REFERENCES}

1. WHO. The Global Burden of Disease: 2004 Update. World Health Organization; Geneva, Switzerland: 2004.

2. Unutzer J, Patrick DL, Simon G, et al. Depressive symptoms and the cost of health services in HMO patients aged 65 years and older. A 4-year prospective study. JAMA. 1997; 277(20):1618-1623. [PubMed: 9168292]

3. Bush DE, Ziegelstein RC, Tayback M, et al. Even minimal symptoms of depression increase mortality risk after acute myocardial infarction. Am J Cardiol. 2001; 88(4):337-341. [PubMed: 11545750]

4. Dauchet L, Amouyel P, Dallongeville J. Fruit and vegetable consumption and risk of stroke: a metaanalysis of cohort studies. Neurology. 2005; 65(8):1193-1197. [PubMed: 16247045]

5. Dauchet L, Amouyel P, Hercberg S, Dallongeville J. Fruit and vegetable consumption and risk of coronary heart disease: a meta-analysis of cohort studies. J Nutr. 2006; 136(10):2588-2593. [PubMed: 16988131]

6. Kodydkova J, Vavrova L, Zeman M, et al. Antioxidative enzymes and increased oxidative stress in depressive women. Clin Biochem. 2009; 42(13-14):1368-1374. [PubMed: 19527700]

7. Maes M, Mihaylova I, Kubera M, Uytterhoeven M, Vrydags N, Bosmans E. Increased 8-hydroxydeoxyguanosine, a marker of oxidative damage to DNA, in major depression and myalgic encephalomyelitis / chronic fatigue syndrome. Neuro Endocrinol Lett. 2009; 30(6):715-722. [PubMed: 20035260]

8. Maes M, Mihaylova I, Kubera M, Uytterhoeven M, Vrydags N, Bosmans E. Increased plasma peroxides and serum oxidized low density lipoprotein antibodies in major depression: markers that further explain the higher incidence of neurodegeneration and coronary artery disease. J Affect Disord. 2010; 125(1-3):287-294. [PubMed: 20083310]

9. Forlenza MJ, Miller GE. Increased serum levels of 8-hydroxy-2' ${ }^{\prime}$-deoxyguanosine in clinical depression. Psychosom Med. 2006; 68(1):1-7. [PubMed: 16449405]

10. Cumurcu BE, Ozyurt H, Etikan I, Demir S, Karlidag R. Total antioxidant capacity and total oxidant status in patients with major depression: impact of antidepressant treatment. Psychiatry Clin Neurosci. 2009; 63(5):639-645. [PubMed: 19674383]

11. Samieri C, Jutand MA, Feart C, Capuron L, Letenneur L, Barberger-Gateau P. Dietary patterns derived by hybrid clustering method in older people: association with cognition, mood, and selfrated health. J Am Diet Assoc. 2008; 108(9):1461-1471. [PubMed: 18755318]

12. Akbaraly TN, Brunner EJ, Ferrie JE, Marmot MG, Kivimaki M, Singh-Manoux A. Dietary pattern and depressive symptoms in middle age. Br J Psychiatry. 2009; 195(5):408-413. [PubMed: 19880930]

13. Park JY, You JS, Chang KJ. Dietary taurine intake, nutrients intake, dietary habits and life stress by depression in Korean female college students: a case-control study. J Biomed Sci. 2010; 17(Suppl 1):S40. [PubMed: 20804617]

14. Tolmunen T, Voutilainen S, Hintikka J, et al. Dietary folate and depressive symptoms are associated in middle-aged Finnish men. J Nutr. 2003; 133(10):3233-3236. [PubMed: 14519816]

15. Payne ME, Jamerson BD, Potocky CF, Ashley-Koch AE, Speer MC, Steffens DC. Natural food folate and late-life depression. J Nutr Elder. 2009; 28:348-358. [PubMed: 21184377]

16. Bjelakovic G, Nikolova D, Gluud LL, Simonetti RG, Gluud C. Mortality in randomized trials of antioxidant supplements for primary and secondary prevention: systematic review and metaanalysis. JAMA. 2007; 297(8):842-857. [PubMed: 17327526]

17. Steffens DC, Welsh-Bohmer KA, Burke JR, et al. Methodology and preliminary results from the neurocognitive outcomes of depression in the elderly study. J Geriatr Psychiatry Neurol. 2004; 17(4):202-211. [PubMed: 15533991] 
18. Robins LN, Helzer JE, Croughan J, Ratcliff KS. National Institute of Mental Health Diagnostic Interview Schedule. Its history, characteristics, and validity. Arch Gen Psychiatry. 1981; 38(4): 381-389. [PubMed: 6260053]

19. Payne ME, Hybels CF, Bales CW, Steffens DC. Vascular nutritional correlates of late-life depression. Am J Geriatr Psychiatry. 2006; 14:787-795. [PubMed: 16943175]

20. Subar AF, Thompson FE, Kipnis V, et al. Comparative validation of the Block, Willett, and National Cancer Institute food frequency questionnaires : the Eating at America's Table Study. Am J Epidemiol. 2001; 154(12):1089-1099. [PubMed: 11744511]

21. Averina M, Nilssen O, Brenn T, Brox J, Arkhipovsky VL, Kalinin AG. Social and lifestyle determinants of depression, anxiety, sleeping disorders and self-evaluated quality of life in Russia--a population-based study in Arkhangelsk. Soc Psychiatry Psychiatr Epidemiol. 2005; 40(7):511-518. [PubMed: 16088370]

22. Harrington J, Perry IJ, Lutomski J, et al. Living longer and feeling better: healthy lifestyle, selfrated health, obesity and depression in Ireland. Eur J Public Health. 2009; 20(1):91-95. [PubMed: 19587230]

23. Sanchez-Villegas A, Delgado-Rodriguez M, Alonso A, et al. Association of the Mediterranean dietary pattern with the incidence of depression: the Seguimiento Universidad de Navarra/ University of Navarra follow-up (SUN) cohort. Arch Gen Psychiatry. 2009; 66(10):1090-1098. [PubMed: 19805699]

24. Jacka FN, Pasco JA, Mykletun A, et al. Association of Western and traditional diets with depression and anxiety in women. Am J Psychiatry. 2010; 167(3):305-311. [PubMed: 20048020]

25. Merrill RM, Taylor P, Aldana SG. Coronary Health Improvement Project (CHIP) is associated with improved nutrient intake and decreased depression. Nutrition. 2008; 24(4):314-321. [PubMed: 18296026]

26. Hamer M, Bates CJ, Mishra GD. Depression, physical function, and risk of mortality: National Diet and Nutrition Survey in adults older than 65 years. Am J Geriatr Psychiatry. 2011; 19(1):7278. [PubMed: 20808095]

27. Craft NE, Haitema TB, Garnett KM, Fitch KA, Dorey CK. Carotenoid, tocopherol, and retinol concentrations in elderly human brain. J Nutr Health Aging. 2004; 8(3):156-162. [PubMed: 15129301]

28. Chang CC, Yu SC, McQuoid DR, et al. Reduction of dorsolateral prefrontal cortex gray matter in late-life depression. Psychiatry Res. 2011; 193(1):1-6. [PubMed: 21596532]

29. Taylor WD, Kuchibhatla M, Payne ME, et al. Frontal white matter anisotropy and antidepressant remission in late-life depression. PLoS ONE. 2008; 3(9):e3267. [PubMed: 18813343]

30. Milaneschi Y, Bandinelli S, Penninx BW, et al. The relationship between plasma carotenoids and depressive symptoms in older persons. World J Biol Psychiatry. 2011

31. Ford DE, Erlinger TP. Depression and C-reactive protein in US adults: data from the Third National Health and Nutrition Examination Survey. Arch Intern Med. 2004; 164(9):1010-1014. [PubMed: 15136311]

32. Chun OK, Floegel A, Chung SJ, Chung CE, Song WO, Koo SI. Estimation of antioxidant intakes from diet and supplements in U.S. adults. J Nutr. 2010; 140(2):317-324. [PubMed: 20032488]

33. Kazes M, Danion JM, Grange D, et al. Eating behaviour and depression before and after antidepressant treatment: a prospective, naturalistic study. J Affect Disord. 1994; 30(3):193-207. [PubMed: 8006246]

34. Anderson SE, Cohen P, Naumova EN, Must A. Association of depression and anxiety disorders with weight change in a prospective community-based study of children followed up into adulthood. Arch Pediatr Adolesc Med. 2006; 160(3):285-291. [PubMed: 16520448] 
Table 1

Participant Characteristics: Sociodemographics, Medical Comorbidity, Body Mass Index and Diet ${ }^{a}$

\begin{tabular}{|c|c|c|c|c|}
\hline & Total $(\mathbf{n}=\mathbf{2 7 8})$ & $\begin{array}{l}\text { Depression } \\
(n=144)\end{array}$ & $\begin{array}{l}\text { Comparison } \\
(\mathbf{n}=134)\end{array}$ & p value $b$ \\
\hline Age in years & $70.4(6.0)$ & $69.5(6.2)$ & $71.3(5.7)$ & 0.02 \\
\hline Sex (female) & $173(62 \%)$ & $78(54 \%)$ & $95(71 \%)$ & 0.004 \\
\hline Race $^{c}$ & & & & 0.2 \\
\hline White & $239(86 \%)$ & $127(88 \%)$ & $112(84 \%)$ & \\
\hline African American & $24(9 \%)$ & $8(6 \%)$ & $16(12 \%)$ & \\
\hline Asian & $7(3 \%)$ & $5(3 \%)$ & $2(1 \%)$ & \\
\hline Native American & $1(0.4 \%)$ & $1(0.7 \%)$ & $0(0 \%)$ & \\
\hline Multiracial & $7(3 \%)$ & $3(2 \%)$ & $4(3 \%)$ & \\
\hline Education in years & $14.8(2.3)$ & $14.3(2.5)$ & $15.3(2.0)$ & 0.0003 \\
\hline Comorbidity score $^{d}$ & $4.9(1.2)$ & $5.2(1.4)$ & $4.5(0.8)$ & $<0.0001$ \\
\hline Body mass index (BMI) & $26.5(4.6)$ & $27.6(4.5)$ & $25.4(4.5)$ & $<0.0001$ \\
\hline Dietary supplement use (yes) ${ }^{e}$ & $200(80 \%)$ & $92(78 \%)$ & $108(82 \%)$ & 0.4 \\
\hline Energy (kcal) & $1710.6(673.2)$ & $1764.1(718.2)$ & $1653.1(618.7)$ & 0.2 \\
\hline Total fat (g) & $66.8(34.9)$ & $72.0(37.7)$ & $61.1(30.8)$ & 0.009 \\
\hline Alcohol (g) & $7.7(12.0)$ & $6.0(11.6)$ & $9.6(12.1)$ & 0.01 \\
\hline \multicolumn{5}{|l|}{${ }_{\text {mean }}(\mathrm{SD})$ or \# $(\%)$} \\
\hline \multicolumn{5}{|c|}{$b$ p value for difference between groups (chi-squared test used to compare proportions; t-test used to compare means). } \\
\hline \multicolumn{5}{|c|}{$c$ percentages do not total $100 \%$ due to rounding. } \\
\hline \multicolumn{5}{|c|}{$d_{\text {comorbid conditions include diabetes, hypertension, arteriosclerosis, and heart disease (score of 1-4 for each condition to yield total score of }}$} \\
\hline
\end{tabular}


Table 2

Dietary Intake of Fruits, Vegetables, and Antioxidants by Participant Group ${ }^{a, b}$

\begin{tabular}{|c|c|c|c|c|}
\hline & $\begin{array}{l}\text { Sample } \\
\mathrm{n}=278\end{array}$ & $\begin{array}{l}\text { Depression } \\
\mathrm{n}=\mathbf{1 4 4}\end{array}$ & $\begin{array}{l}\text { Comparison } \\
\mathbf{n}=134\end{array}$ & p value ${ }^{c}$ \\
\hline Fruits (svgs/d) & $1.9(1.2)$ & $1.6(1.2)$ & $2.2(1.1)$ & $<0.0001$ \\
\hline Vegetables (svgs/d) & $3.2(1.8)$ & $3.0(1.7)$ & $3.4(1.9)$ & 0.03 \\
\hline \multicolumn{5}{|l|}{ Vitamin C (mg/d) } \\
\hline dietary & $128.0(66.7)$ & $109.2(62.2)$ & $148.3(65.6)$ & $<0.0001$ \\
\hline supplemental & $309.2(494.7)$ & $290.9(460.2)$ & $328.8(530.3)$ & 0.5 \\
\hline total & $437.2(503.8)$ & $400.0(464.1)$ & $477.1(542.2)$ & 0.2 \\
\hline \multicolumn{5}{|l|}{ Vitamin E (mg/d) } \\
\hline \multicolumn{5}{|l|}{ alpha-tocopherol } \\
\hline dietary & $10.0(5.3)$ & $9.9(5.2)$ & $10.2(5.4)$ & 0.7 \\
\hline supplemental & $151.5(175.8)$ & $146.1(184.4)$ & $157.3(166.6)$ & 0.6 \\
\hline total & $161.5(176.1)$ & $156.0(184.5)$ & $167.5(167.1)$ & 0.6 \\
\hline gamma-tocopherol $^{d}$ & $22.0(13.4)$ & $23.3(15.0)$ & $20.6(11.4)$ & 0.1 \\
\hline Selenium $(\mu \mathrm{g} / \mathrm{d})^{e}$ & $21.1(44.6)$ & $22.6(48.0)$ & $19.4(40.7)$ & 0.5 \\
\hline Carotenoids ( $\mu \mathrm{g} / \mathrm{d})$ & $11225.2(7502.9)$ & $10699.9(7334.3)$ & $11789.7(7667.3)$ & 0.2 \\
\hline $\begin{array}{l}\text { alpha-carotene } \\
\text { beta-carotene }\end{array}$ & $820.3(766.6)$ & $751.8(737.0)$ & $893.8(793.3)$ & 0.1 \\
\hline dietary & $3941.1(2860.2)$ & $3759.0(2937.5)$ & $4136.7(2772.4)$ & 0.3 \\
\hline supplemental & $1974.8(5288.6)$ & 2036.7 (5532.9) & $1908.2(5032.8)$ & 0.8 \\
\hline total & $5915.8(6431.4)$ & $5795.7(6889.2)$ & $6044.9(5923.3)$ & 0.7 \\
\hline beta-cryptoxanthin & $172.6(139.0)$ & $140.0(137.8)$ & $207.7(132.1)$ & $<0.0001$ \\
\hline lutein & $1903.2(1682.4)$ & $1681.9(1416.2)$ & $2141.0(1904.9)$ & 0.02 \\
\hline lycopene & $4388.0(4890.1)$ & $4367.1(5061.8)$ & $4410.5(4717.4)$ & 0.9 \\
\hline \multicolumn{5}{|l|}{${ }^{a}$ mean $(\mathrm{SD})$} \\
\hline \multicolumn{5}{|c|}{$b_{\text {Intake estimates derived from } 1998 \text { Block food frequency questionnaire (NutritionQuest; Berkele }}$} \\
\hline
\end{tabular}


Table 3

Fruit, Vegetable, and Antioxidant Logistic Regression Models for Depression ${ }^{a, b}$

\begin{tabular}{|c|c|c|c|}
\hline & Estimate & $\chi^{2}$ & p value \\
\hline Fruits (svgs/d) & $0.26(0.13)$ & 4.3 & 0.038 \\
\hline Vegetables (svgs/d) & $0.16(0.08)$ & 4.0 & 0.047 \\
\hline \multicolumn{4}{|l|}{ Vitamin C (mg/d) } \\
\hline dietary & $0.0083(0.0024)$ & 12.3 & 0.0005 \\
\hline supplemental & $0.000078(0.00027)$ & 0.08 & 0.8 \\
\hline total & $0.00020(0.00027)$ & 0.53 & 0.5 \\
\hline \multicolumn{4}{|l|}{ Vitamin E (mg/d) } \\
\hline $\operatorname{dietary}^{c}$ & $0.027(0.017)$ & 2.47 & 0.12 \\
\hline supplemental $^{d}$ & $-0.00030(0.00079)$ & 0.15 & 0.7 \\
\hline total & $-0.00024(0.00079)$ & 0.10 & 0.8 \\
\hline Selenium $(\mu \mathrm{g} / \mathrm{d})^{e}$ & $-0.0045(0.0031)$ & 2.12 & 0.15 \\
\hline \multicolumn{4}{|l|}{ Carotenoids $(\mu \mathrm{g} / \mathrm{d})$} \\
\hline alpha-carotene & $0.00016(0.00018)$ & 0.78 & 0.4 \\
\hline \multicolumn{4}{|l|}{ beta-carotene } \\
\hline dietary & $0.000030(0.000049)$ & 0.38 & 0.5 \\
\hline supplemental & $-0.000018(0.000025)$ & 0.55 & 0.5 \\
\hline total & $-0.0000074(0.000021)$ & 0.13 & 0.7 \\
\hline beta-cryptoxanthin & $0.0028(0.0010)$ & 7.3 & 0.007 \\
\hline lutein & $0.00013(0.000093)$ & 2.0 & 0.2 \\
\hline lycopene & $0.000016(0.000029)$ & 0.3 & 0.6 \\
\hline
\end{tabular}

'Separate logistic regression models ( $\mathrm{df}=1$ for all variables); values are mean (standard error); models controlled for age, sex, race, education, comorbidity score, BMI, total fat, and alcohol intake.

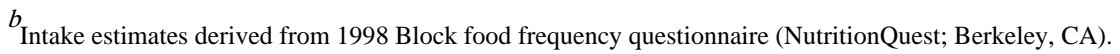

c alpha- and gamma-tocopherol.

dalpha-tocopherol only.

$e_{\text {supplemental sources only. }}$ 